\title{
Una propuesta metodológica para elaborar videos creativos en clase de geometría
}

\section{A methodological proposal to design creative videos in geometry class}

DOI: http://dx.doi.org/10.17981/cultedusoc.12.2.2021.05

Recibido: 27 de agosto de 2020. Aceptado: 10 de diciembre de 2020. Publicado: 1 de julio de 2021.

Jonathan Cervantes-Barraza (i)

Universidad del Atlántico. Barranquilla (Colombia)

jacervantes@mail.uniatlantico.edu.co

Para citar este artículo:

Cervantes-Barraza, J. (2021). Una propuesta metodológica para elaborar videos creativos en clase de geometría. Cultura, Educación y Sociedad, 12(2), 79-94. DOI: http://dx.doi.org/10.17981/cultedusoc.12.2.2021.05

\section{Resumen}

En el marco de la pandemia producida por el virus SARS-coV-2 se provee una propuesta metodológica para elaborar videos creativos de geometría como un medio o herramienta que permita abordar la enseñanza de conceptos de la geometría Euclidiana. Los videos son producto de seminarios de investigación con futuros profesores de matemáticas donde se aborda la enseñanza de la geometría desde enfoques basados en la construcción de conocimiento matemático y los Procesos de Formación de Conceptos (PFC). En la investigación se implementó una metodología de corte cualitativa y descriptiva que permitió analizar el contenido matemático de los videos, los materiales y las actividades propuestas por los profesores de matemáticas en formación. El principal resultado de la investigación es una propuesta metodológica para la elaboración de Videos Creativos de Geometría (VCG), esta proporciona un conjunto de principios que permiten a los profesores de matemáticas diseñar sus propios videos creativos con el fin de mejorar la enseñanza de la geometría apoyado del proceso de formación de conceptos y generar espacios de reflexión en cuanto a medios digitales abiertos que apoyen los procesos de enseñanza de la geometría en tiempos de pandemia.

Palabras clave: Videos; Matemáticas; Geometría; Metodología

\begin{abstract}
In the framework of the pandemic produced by the SARS-coV-2 virus, a methodological proposal is provided to elaborate creative geometry videos as a means or tool that allows to approach the teaching of concepts of Euclidean geometry. The videos are the product of research seminars with future mathematics teachers where the teaching of geometry is approached from approaches based on the construction of mathematical knowledge and Concept Formation Processes (PFC). In the research, a qualitative and descriptive methodology was implemented that allowed us to analyze the mathematical content of the videos, materials and activities proposed by the mathematics teachers in training. The main result of the research is a methodological proposal for the elaboration of Creative Videos of Geometry (VCG), this provides a set of principles that allow mathematics teachers to design their own creative videos in order to improve the teaching of geometry supported by the process of concept formation and generating spaces for reflection in terms of open digital media that support the teaching processes of geometry in times of pandemic
\end{abstract}

Keywords: Videos; Mathematics; Geometry; Methodology 


\section{INTRODUCCIÓN}

El sistema educativo en América latina y en diversos países del mundo ha sido afectado por la pandemia mundial producida por el coronavirus COVID-19 (SARS-coV-2), implicando el confinamiento de miles de personas, cierre de comercio, empresas, centros educativos. En respuesta a la emergencia sanitaria, el gobierno de Colombia, decidió suspender las clases presenciales en todas las instituciones educativas e implementar estrategias de enseñanza y aprendizaje virtual (Moreno-Correa, 2020; Decreto 749, 2020; Decreto 660, 2020). Cabe destacar que son múltiples los factores que afectan el desarrollo de los procesos de enseñanza y aprendizaje basado en la virtualidad, por ejemplo, la brecha tecnológica (Francesc, 2020). Lo anterior se fundamenta en documentos oficiales del Instituto Internacional de la UNESCO para la Educación Superior en América Latina y el Caribe (IESALC, 2020), dado que el cierre temporal de las instituciones de educación superior ha afectado aproximadamente a unos 23,4 millones de estudiantes de educación superior y a 1.4 millones de docentes en América Latina y el Caribe.

El sector educativo y los niveles escolares desde educación escolar hasta el nivel superior se ha visto afectado en la falta de recursos tecnológicos para abordar metodologías propuestas en la situación sanitaria que atraviesa el país. En este contexto, autores como MorenoCorrea (2020) señalan que "se hace cada vez más importante la formación de los docentes en estrategias de educación innovadoras, el uso pedagógico de las TICS (Tecnologías de la Información y de la Comunicación)” (p. 15). Por ello, en el presente artículo se pretende contribuir a analizar el impacto de la pandemia sobre el sistema educativo y los procesos de enseñanza aprendizaje a través de una propuesta metodológica que permita al profesor de matemáticas crear sus propios videos de clase.

La incorporación de la tecnología de la información y comunicación en la educación se considera una de las bases fundamentales para el óptimo desarrollo de las competencias tecnológicas de los estudiantes y éstas a la vez permitirán hacer frente a un mundo globalizado (Morales, Guzmán y Tecnología Educativa, 2015). Esto ha sido posible gracias al desarrollo de técnicas y metodologías de procesamiento muy efectivas para la visualización gráfica, llegando a permear el campo de la educación a través de la creación de nuevas herramientas de difusión, de estructuras para la creación de conocimiento y aprendizaje (Ballesteros-Ballesteros, Rodríguez-Cardoso y Lozano-Forero, 2021).

La implementación de herramientas audiovisuales con fines educativos se ha generalizado en las últimas décadas, entre ellas, el vídeo educativo es una de las más destacadas (De la Fuente, Hernández y Pra, 2012; Burgos, Beltrán-Pellicer y Godino, 2020). Los videos educativos favorecen el proceso de enseñanza y aprendizaje de la matemática, ayudan a superar dificultades de los estudiantes y aumentan el interés por aprender matemáticas (Velasco, Montiel y Ramírez, 2018). En el marco de la enseñanza de la matemática, Velasco et al. (2018) sostienen que el uso de los videos educativos apoya el proceso de aprendizaje de los estudiantes, mientras que Morales et al. (2015) señalan que es un recurso que impacta audiovisualmente y permite la transmisión de información con base en animaciones coloridas, música y demás. En este sentido, utilizar herramientas audiovisuales con fines educativos permite abordar la enseñanza y el aprendizaje de la geometría al tener en cuenta la influencia de las formas de presentar la información gráfica en la comprensión de los estudiantes (Gutiérrez y Jaime, 2012). 
En el marco de la enseñanza y aprendizaje de la geometría, se han documentado problemas de corte didáctico y pedagógico que han implicado el escaso desarrollo de la geometría en el currículo escolar implementado en la educación básica, así como lo señala Camargo, Samper y Perry (2018) "se limitó la enseñanza a contenidos sobre algunos conceptos geométricos, clasificación y fórmulas para calcular el área y el perímetro de figuras dejando de lado la enseñanza de la demostración” (p. 176). Esta problemática implica el contenido matemático y cómo se enseñan conceptos de la geometría a los estudiantes pasando por alto procesos de que permiten construir un concepto geométrico (e.g., análisis, abstracción, generalización y síntesis). Aunado a esto, se reconocen diversas ventajas de abordar el estudio de la geometría Euclidiana, así como lo afirma Moreno-Armella y Elizondo (2017), la geometría euclidiana permite analizar y comprender el espacio físico que nos rodea y relacionarlo con elementos básicos de la geometría como rectas, puntos, planos, además abordar su estudio favorece el desarrollo de competencias relacionadas con el sentido espacial y el razonamiento matemático de los estudiantes (Camargo et al., 2018).

En aras de abordar la problemática planteada con respecto a la enseñanza de conceptos base de la geometría Euclidiana, en esta investigación se recurre al uso de videos creativos como una herramienta educativa que permite al estudiante construir los conceptos geométricos basados en actividades de corte práctico y teórico. En apoyo a las ideas anteriores, Sandoval, Cagua, Álvarez, Molina y Estrada (2018) aseguran que "el uso de vídeos teóricos y explicativos aclaran conceptos, definiciones y enunciados" (p. 1), los estudiantes consideran que es una buena herramienta de aprendizaje, tener vídeos sobre las partes más importantes de la clase, especialmente en la resolución de ejercicios y gran parte de ellos le dedicaría un tiempo mayor a 10 minutos para visualizar y complementar su formación académica

La investigación se desarrolla en el contexto de la educación virtual, medio que permite la trasmisión de información para la construcción del conocimiento por parte de los estudiantes; esto podría ayudar a mejorar su entendimiento (Moreno-Correa, 2020). Son diversos las herramientas tecnológicas implementadas para mediar la situación de contingencia que imposibilita realizar procesos de enseñanza en lo presencial. En este sentido, De la Fuente et al. (2012), reconocen que "de las nuevas tecnologías TIC, en general, el vídeo en particular, es indiscutible a la hora de hacer más atractivo el aprendizaje y la enseñanza en el siglo XXI" (p. 188).

El video se ha implementado en los últimos años como un recurso didáctico en apoyo al proceso de enseñanza y aprendizaje de la matemática. Existen tipos de videos en el contexto educativo, por mencionar algunos, De la Fuente et al. (2012) definen el vídeo educativo como "un medio didáctico que facilita el desarrollo de un proceso de aprendizaje y destaca los cuatro componentes que lo conforman: Un soporte material, un contenido, una forma simbólica de representar la información, una finalidad o propósito educativo" (p.178). Investigaciones donde se aborda el concepto de videos didácticos y los minivideos, lo conciben como un recurso audiovisual que se ajusta a los requerimientos del docente y sirve de apoyo en su práctica educativa (Morales et al., 2015). En este artículo se recurre al término videos creativos con el fin de tratar el aprendizaje desde la interacción con un medio y objetos que permitan al estudiante construir su propio conocimiento. 
En el artículo se concibe la problemática educativa que implica: 1) El distanciamiento social producto de la emergencia sanitaria que ha permeado en el desarrollo del año escolar basado en la virtualidad, generando un cambio de estrategias para abordar la enseñanza de la matemática y de la geometría. 2) La necesidad de abordar contenidos matemáticos con base en recursos tecnológicos como los videos educativos que involucren a los estudiantes en el aprendizaje. 3) Los videos educativos son un recurso digital abierto que permite al estudiante construir conocimientos basados en la visualización, la práctica y la imitación. En efecto, esta investigación tiene como objetivo principal diseñar una propuesta metodológica para elaborar videos creativos de geometría con base en los procesos de formación de conceptos, análisis, abstracción, generalización y síntesis. Es decir, proporcionar una herramienta que permita a los profesores diseñar videos de clases prácticas con implicaciones en análisis de materiales concretos con formas geométricas.

\section{Metodología}

El estudio implicó un enfoque de investigación cualitativo (Hernández, Fernández y Baptista, 2014), aborda el diseño de videos educativos y se fundamenta en la adaptación de la propuesta metodológica para elaborar videos educativos diseñada por Sandoval et al. (2018). La adaptación de la propuesta metodológica se desarrolló con base en los principios de adaptación: identificación, transferencia y ajustes de la aplicación del método seleccionado (Braun \& Lindeman, 2004). Se identificó la propuesta del diseño de videos educativos y se observó que no tienen en cuenta los procesos de formación de conceptos, esta una razón para implementar los elementos constitutivos de la propuesta y ajustarla de tal forma que se optimice en el diseño de videos con base en materiales concretos.

\section{Materiales y participantes}

Los videos creativos son producto de seminarios de investigación sobre la enseñanza de la geometría Euclidiana, en el cual participaron 75 profesores de matemáticas en formación matriculados en una universidad pública ubicada en la costa Atlántica de Colombia. El seminario impartido por el autor de este artículo, los profesores en formación recibieron orientaciones teóricas sobre el proceso de formación de conceptos en el marco de la geometría, motivándolos en la construcción de propuestas innovadoras para transmitir conocimiento matemático al aplicar la propuesta metodológica presentada en esta investigación. Se realizaron cinco sesiones, cada sesión con una hora y media de duración requirió a los profesores en formación conectarse por medio de video llamadas de Google. En las primeras dos sesiones se presentaron los fundamentos teóricos de la propuesta metodológica, en la tercera y cuarto los profesores planearon el diseño de los videos creativo y luego en una quinta sesión compartieron la experiencia con el objetivo de reflexionar sobre los videos filmados. 


\section{Fundamentos de la propuesta metodológica}

El concepto de video creativo es un recurso educativo abierto que permite transmitir conocimiento relacionado con la geometría a través de un conjunto de actividades de corte práctico (e.g., recortar, medir, calcular, dibujar, por mencionar algunas) e involucra al estudiante en el aprendizaje del contenido matemático que se aborde. Los videos se denominan creativos porque involucran al estudiante en la experimentación de situaciones concretas que implican conceptos de la geometría, por ejemplo, usar una tapa de gaseosa para determinar el valor del número, una vez el estudiante obtenga el cociente entre la longitud de la circunferencia y el diámetro de la tapa. El objetivo de los videos creativos es involucrar al estudiante con su medio y evidenciar la relación que tienen los objetos del mudo con las matemáticas, en particular en el contexto de la geometría.

La geometría se considera como el producto de la estrecha relación con otros dominios matemáticos y la vida cotidiana (Camargo y Acosta, 2012), su enseñanza debe ir alineada con el proceso de construcción de conocimiento teórico el cual inicia desde lo sensitivo hasta lo abstracto (Díaz, 2007). El conocimiento desde una perspectiva del materialismo de la teoría del conocimiento se constituye en: conocimiento sensitivo o material y conocimiento teórico. El primero es producto de los reflejos directos de los objetos (realidad) sobre la conciencia de las personas, en este proceso de construcción intervienen la sensación, la noción, y la percepción. En cambio, en la construcción de conocimiento teórico es producto de los reflejos indirectos de la realidad subjetiva sobre la conciencia del hombre y requiere de actividades cognitivas que involucran el concepto, juicio y razonamiento.

En el marco de la construcción de conocimiento teórico, el autor señala que el Proceso de Formación de Conceptos (PFC) requiere de cuatro actividades cognoscitivas: analizar, abstrae, generalizar y sintetizar (Díaz, 2007). El análisis refiere a la identificación y descripción de las características invariantes y comunes de los objetos matemáticos, la abstracción consiste en depurar las características identificadas que no definen el concepto matemático, la generalización implica atribuir las características invariantes identificadas a otros objetos matemáticos que refieren al concepto en estudio. Y la síntesis es producto de reunir las propiedades y característica invariantes que definen el concepto matemático.

El contenido de los videos creativos se fundamenta en la correspondencia entre las actividades: analizar, abstraer, generalizar y sintetizar realizadas en el Proceso de Formación de Concepto (PFC) y cuatro momentos que constituyen la duración de un video (Figura 1). El momento inicial se corresponde con la actividad de análisis, el estudiante se dispone a realizar la identificación y descripción de las características de casos particulares del objeto matemático en estudio (e.g., cuadrado, triángulo, esfera, entre otros). El momento intermedio 1 implica la actividad de abstracción y demanda al estudiante realizar una depuración de todas las características identificadas en el momento anterior con el fin de seleccionar las características esenciales que constituyen el objeto matemático. En el momento intermedio 2 se relaciona con la generalización, en este momento el estudiante extiende las características invariantes o esenciales abstraídas en el momento anterior de todos los objetos matemáticos que guardan similitud con el objeto matemático en estudio. 
Y el momento final se relaciona con la síntesis, el estudiante realiza una composición articulada de las características invariantes que cumple el objeto matemático analizado en términos de una definición o enunciado (Figura 1).

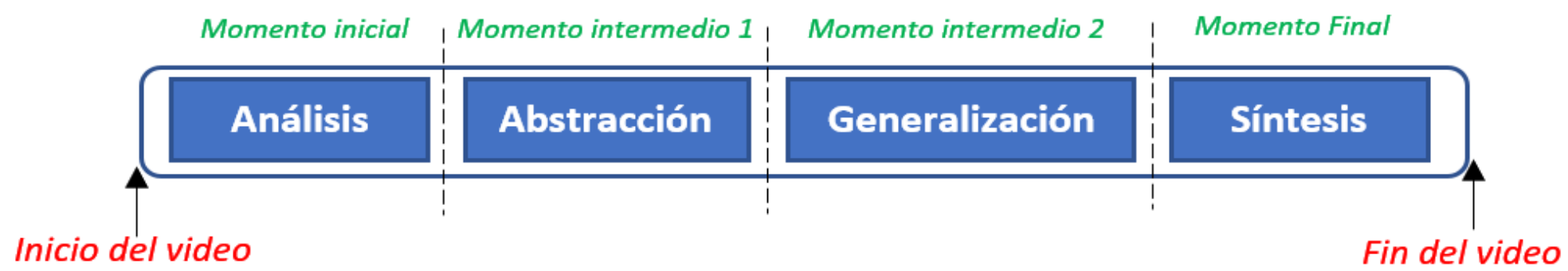

Figura 1. Correspondencia entre las actividades del proceso de formación de concepto (PFC) y los momentos que constituyen la duración de un video creativo. Fuente: Elaboración propia.

\section{Fases de la propuesta metodológica}

La propuesta metodológica Videos Creativos de Geometría (VCG) que se plantea en esta investigación es una adaptación de la metodológica para elaborar videos educativos diseñada por Sandoval et al. (2018). Esta se constituye de 8 etapas que indican al profesor cómo elaborar un video desde la elección de la temática hasta la puesta en escena en el salón de clase. La adaptación de esta propuesta metodológica es alineada con el objetivo de los videos creativos, involucrar al estudiante con su medio y evidenciar la relación que tienen los objetos del mudo con las matemáticas. Bajo esta perspectiva, el valor agregado de la propuesta metodológica VCG en este artículo consta de integrar los Procesos de Formación de Conceptos (PFC) con los cuatro momentos descriptores de un video creativo con el fin de generar espacios de aprendizaje basado en el uso de materiales concretos. En esta investigación se aborda el concepto de materiales concretos no son simplemente objetos o enseres con una funcionalidad fija y definida (Moreno, 2015), sino como un conjunto de objetos que pueden utilizarse por mediar el proceso de aprendizaje y permitir a los estudiantes reconocer características del objeto matemático.

La metodología (VCG) es una herramienta que le permite al profesor de matemáticas diseñar videos creativos con base en materiales concretos (e.g., tapas, cartulinas, cuerdas, reglas, objetos con forma circular, entre otros) con la finalidad de construir conocimiento empírico en el marco de la geometría. La propuesta metodológica requiere de tres fases (F): planeación (F1), diseño (F2) y ejecución (F3) (Tabla 1). Cada fase demanda al profesor realizar una serie de actividades o pasos $(\mathrm{P})$ que le permitirán diseñar un video creativo. 
TABLA 1.

Fases y pasos de la metodología Videos Creativos en Geometría.

\begin{tabular}{ll}
\hline Fases & \multicolumn{1}{c}{ Pasos } \\
\hline & P1: Selección del contenido matemático \\
& P2: Identificación de material tangibles afines a la temática \\
& P3: Trazar el objetivo del video \\
& P4: Asignación de actividades para cada momento del video \\
& P5: Construcción del guion del video \\
& P6: Grabación del video \\
& P7: Someter a revisión por experto \\
& P8: Rediseño del video \\
& P9: Puesta en escena con los estudiantes \\
& P10: Retroalimentación por parte de los estudiantes
\end{tabular}

Fuente: Elaboración propia.

En la primera fase el profesor selecciona el contenido matemático a tratar en el video (P1), esta selección se realiza con base en el objetivo de aprendizaje. En atención a la temática seleccionada, se deben identificar objetos o material concretos (e.g., cuadro de madera, tapas de plástico, cartulinas, cuerdas, entre otros) que puedan conseguir los estudiantes y que guarden similitud con el objeto geométrico a estudiar (P2). El objetivo de aprendizaje del video se traza con base en el contenido geométrico a tratar y con esto evidenciar los conocimientos que alcanzarán los estudiantes luego de visualizar el video y practicar lo solicitado por el profesor (P3).

Con base en los cuatro momentos que constituyen el video, inicial, intermedio 1, intermedio 2 y final, el profesor debe proponer actividades que refieren al proceso de formación de concepto (P4), actividades que demanden al estudiante analizar las características y propiedades de los objetos geométricos en estudio, realizar procesos de abstracción sobre las características que son imprescindibles y definen al objeto, esto con esto fin de que pueda generalizarlas a otros objetos, y con esto, los estudiantes puedan emitir una síntesis en términos de una definición. El último paso de esta fase demanda al profesor la escritura del guion o libreto que usará para la grabación del video (P5).

La segunda fase refiere al diseño del video, se realizan las primeras grabaciones con base en guion construido (P6). Una vez se cuente con la grabación del video, se dispone a compartir con un experto en la temática de la geometría, un académico que pueda emitir una retroalimentación con el propósito de mejorar cuestiones de fondo y de estructura (P7). El rediseño del video se fija en la retroalimentación que indique el experto, se realizan las respectivas modificaciones al guion para hacer una nueva grabación de video creativo (P8).

La tercera fase tiene que ver con el espacio académico que se genera al compartir el video creativo por medio de las redes sociales o plataformas educativas por medio de la cual se tiene comunicación con los estudiantes. La puesta en escena del video toma lugar en la clase de matemáticas/geometría con el objetivo de iniciar una temática o recurrir a ella con fines de reforzar los conocimientos adquiridos (P9). Una vez los estudiantes vean el video, estos podrán emitir un comentario sobre el video y las actividades requeridas (P10). Se busca con esta retroalimentación de parte de los estudiantes y mejora de los videos creativos. 


\section{Resultados y AnÁlisis}

Los videos creativos de geometría están clasificados por temáticas: figuras geométricas, cuerpos geométricos, ángulos y rectas, área y perímetro, tienen una duración no mayor a diez minutos y están disponibles en una página web interactiva creada por el autor de esta investigación con el propósito de difundir los videos creativos a la comunidad académica (Figura 2), incluyendo colegios privados y públicos pertenecientes a la costa Atlántica, en particular en los municipios del Atlántico. Los videos se pueden visualizar ingresando en el siguiente enlace:

https://view.genial.ly/5f30a8c10c238a0d9c2281f6/learning-experience-didactic-unit-videos-creativos-de-geometria

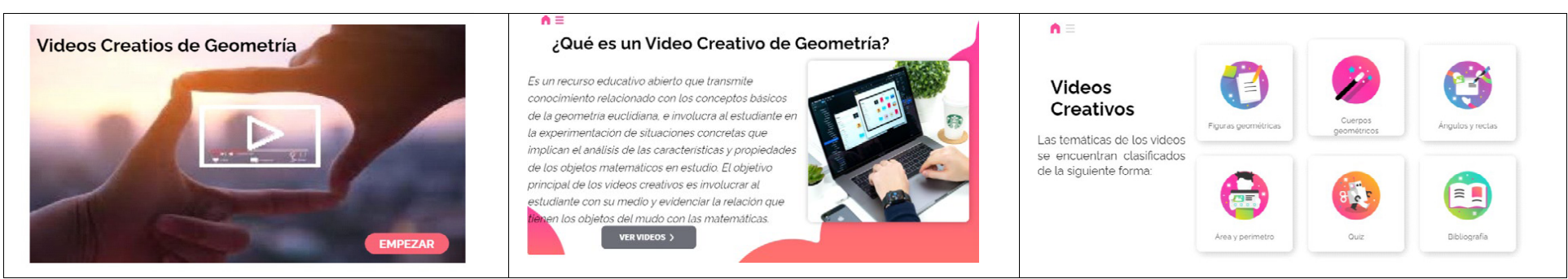

Figura 2. Fotografías de la página web interactiva que contiene los videos creativos de geometría. Fuente: Elaboración propia.

Se presentan algunas fotografías de escenas de los videos creativos de geometría diseñados por un grupo de futuros profesores de matemáticas, quienes recurren a materiales concretos como se puede ver en las Figura 3 y Figura 4. Para la temática del video de la Figura 3, la profesora participante implementó materiales como cartulinas recortadas en forma cuadrada, conos, guirnaldas navideñas para representar los objetos matemáticos: cuadrado, cono y la esfera, respectivamente.

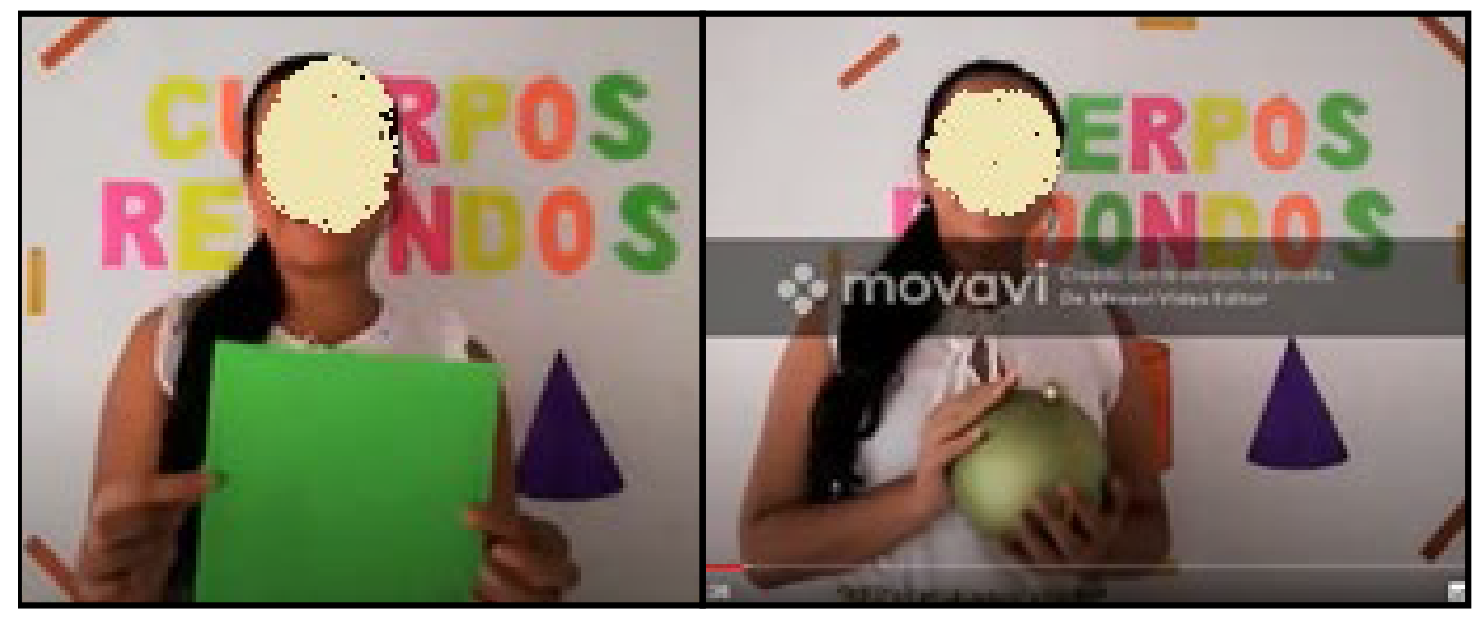

Figura 3. Video creativo sobre los cuerpos redondos.

Fuente: Elaboración propia.

En la Figura 4 se aborda la temática “semejanza de polígonos” y el profesor en formación implementó materiales concretos de forma rectangular como carcasas de celular, tarjetas plásticas de identificación con el objetivo de comparar características de los objetos presentados e introducir la temática de semejanza de rectángulos. 


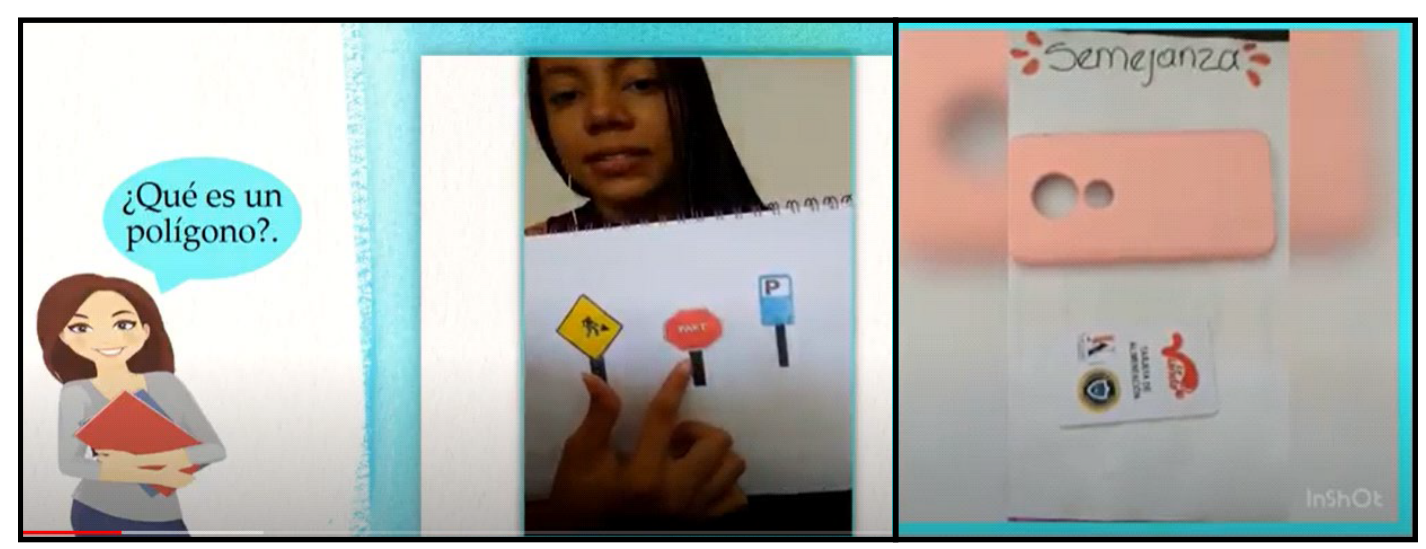

Figura 4. Video creativo sobre la semejanza de polígonos.

Fuente: Elaboración propia.

De forma resumida se presenta en la Tabla 2 una lista de los materiales concretos implementados por los futuros profesores de matemáticas en la elaboración de los videos creativos de distintas temáticas.

TABLA 2.

Materiales concretos implementados para los videos creativos de geometría.

\begin{tabular}{lllll}
\hline $\begin{array}{l}\text { Cuadrado- } \\
\text { rectángulo }\end{array}$ & \multicolumn{1}{c}{ Círculo } & Circunferencia & Triángulos & Prismas \\
\hline $\begin{array}{l}\text { Cartulina con } \\
\text { forma cuadrada }\end{array}$ & Platos & $\begin{array}{l}\text { Cuerda ubicada en el } \\
\text { borde de objetos circulares }\end{array}$ & $\begin{array}{l}\text { Recortados con } \\
\text { cartulina }\end{array}$ & Cajas de cartón \\
$\begin{array}{l}\text { Cuadros o } \\
\text { fotografías }\end{array}$ & $\begin{array}{l}\text { Reloj con forma } \\
\text { circular }\end{array}$ & $\begin{array}{l}\text { Lana ubicada en el borde } \\
\text { de objetos circulares }\end{array}$ & $\begin{array}{l}\text { Objetos con forma } \\
\text { triangular }\end{array}$ & Cubos de Rubik \\
$\begin{array}{l}\text { Cuadernos } \\
\text { Baldosas }\end{array}$ & $\begin{array}{l}\text { Tapas de } \\
\text { gaseosa }\end{array}$ & & & $\begin{array}{l}\text { Objetos con forma } \\
\text { de cilindros }\end{array}$ \\
\hline
\end{tabular}

Fuente: Elaboración propia.

\section{Análisis del contenido de los videos creativos de geometría}

El análisis de los videos producidos por los profesores de matemáticas en formación se realizó con base en una adaptación del modelo de análisis de videos coherente con el proceso de construcción de conocimiento matemático planteado por Planas (2006). Este consiste en seis fases: 1) estudio y descripción general del video, 2) identificación de episodios de revisión de significados, 3) búsqueda de procesos de reelaboración de significados, 4) caracterización de interacciones sociales, 5) elaboración de historias explicativas, y 6) comparación de historias.

La adaptación consistió en retomar las fases 1, 2 y 5 y fundamentar el proceso de análisis de los videos creativos, no se consideraron las fases 3,4 y 6 dado que los videos creativos diseñados por los futuros profesores no presentan episodios de interacción entre profesor y estudiantes. Sin embargo, se retoman elementos que permiten a los investigadoresprofesores realizar un análisis de los videos creativos con base en: a) identificación del contenido matemático, objetivo y materiales implementados en el video, b) descripción del contenido de videos con el propósito de identificar las actividades propuestas en cada 
uno de los procesos de formación del concepto en estudio (Figura 5) y c) presentar una narrativa que resuma el contenido del video en función del objeto matemático, materiales y actividades propuestas. Los elementos mencionados conforman las categorías y permiten caracterizar las actividades propuestas en los videos de los profesores en el marco del proceso de formación de conceptos ver tablas 3, 4 y 5. Los videos diseñados y analizados son evidencia de cómo los futuros profesores de matemáticas implementan materiales concretos para recrear situaciones matemáticas que involucren a los estudiantes en la construcción de conceptos. El contenido de los videos además requiere que los estudiantes implementen analicen las medidas, formas y demás características invariantes que tienen los objetos matemáticos con el fin de establecer una conjetura e identificar rasgos esenciales, propiedades matemáticas.

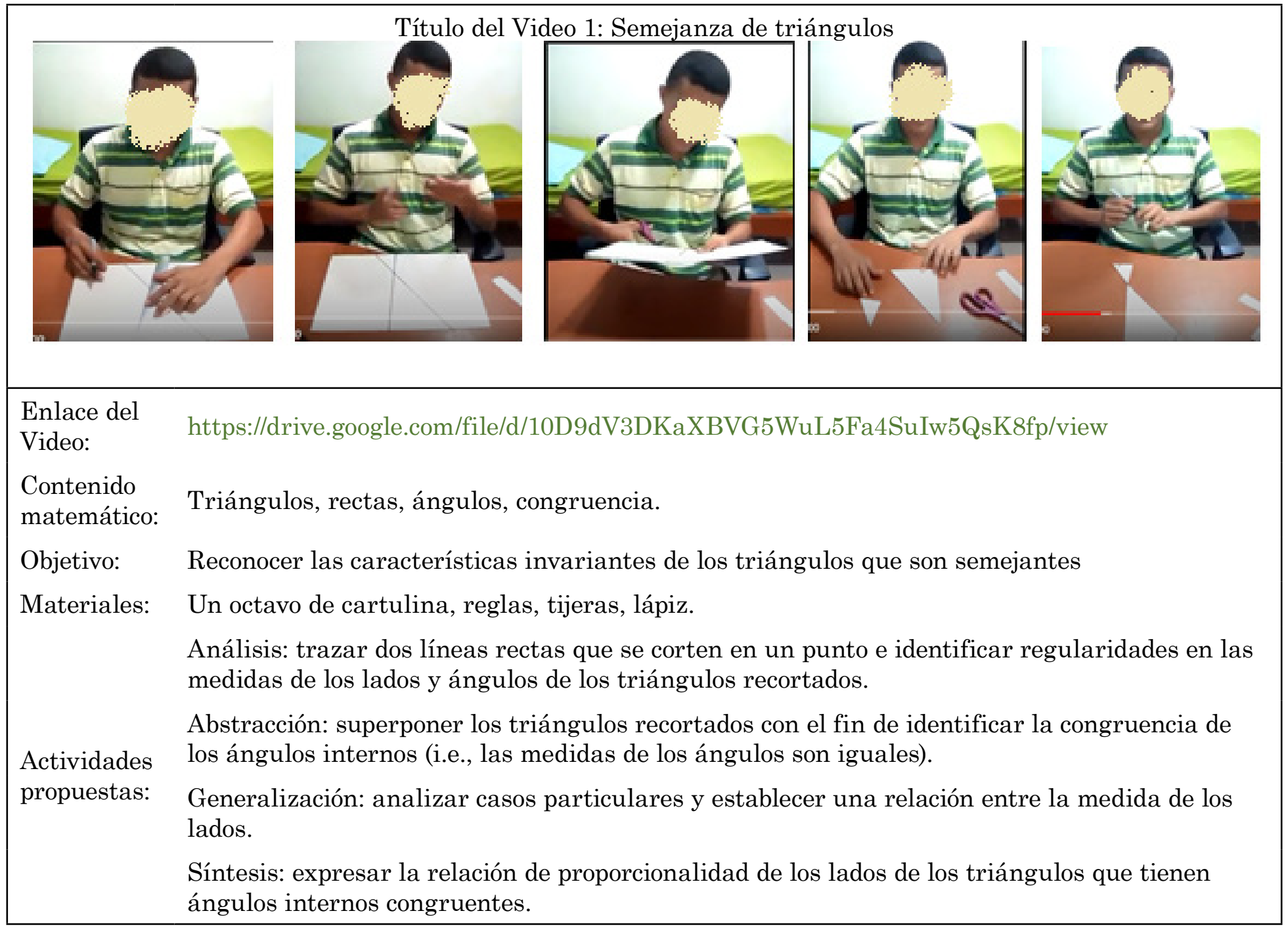

Figura 5. Descripción del contenido del video creativo de geometría: semejanza de triángulos. Fuente: Elaboración propia.

El video creativo de geometría descrito en la Figura 5 aborda la temática de la semejanza de triángulos, el profesor en formación recurre a los siguientes materiales: cartulina, regla y lápices de colores para reconstruir una situación que le permita evidenciar a los estudiantes las características de los triángulos semejantes. Las actividades propuestas en el video conducen al estudiante: analizar, abstraer y generalizar las características invariantes de los triángulos semejantes, el video permite a los estudiantes reconocer la congruencia de los ángulos internos de los triángulos cuando son semejantes e identificar 
la relación de proporcionalidad que existe entre las medidas de los lados de los triángulos. En contraste con el objetivo de la investigación, la metodología VCG incorporó elementos teóricos con actividades de corte práctico para que los estudiantes pudieran establecer relaciones entre los objetos concretos que lo rodean y las propiedades fundamentales de los objetos geométricos.

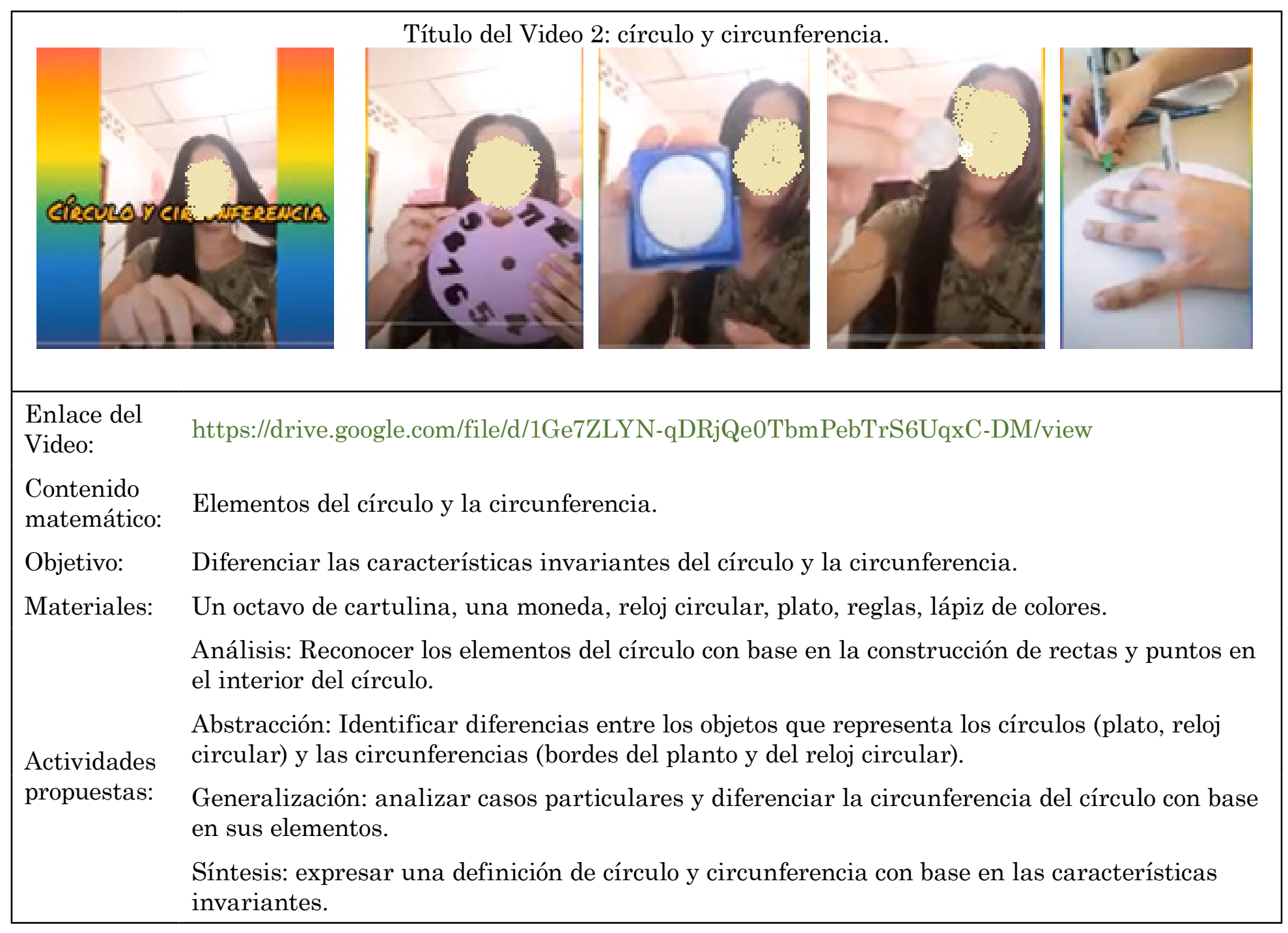

Figura 6. Descripción del contenido del video creativo de geometría: círculo y circunferencia. Fuente: Elaboración propia.

El video creativo de geometría que se presenta en la Figura 6 refiere al contenido matemático "círculo y circunferencia", se diseñó con base en materiales tales como: monedas, platos, reloj con forma circular, cartulina, lápices de colores, reglas con el objetivo de presentar situaciones que involucren a los estudiantes identificar los elementos del círculo y la circunferencia. Así también, las actividades propuestas en el video solicitan al estudiante analizar, abstraer y generalizar que la circunferencia y el círculo son figuras geométricas diferentes que no se pueden confundir. Los materiales implementados para este video son de gran apoyo para que los estudiantes establezcan diferencias en las características de los objetos matemáticos estudiados. En contraste con el proceso de formación de conceptos, Díaz (2007) señala que la construcción de conocimiento empírico tiene sus primemos acercamientos desde el análisis de las características o rasgos de los objetos. Ante esta postura, el análisis de los objetos con forma circular permite a los estudiantes distinguir una circunferencia de un círculo. 


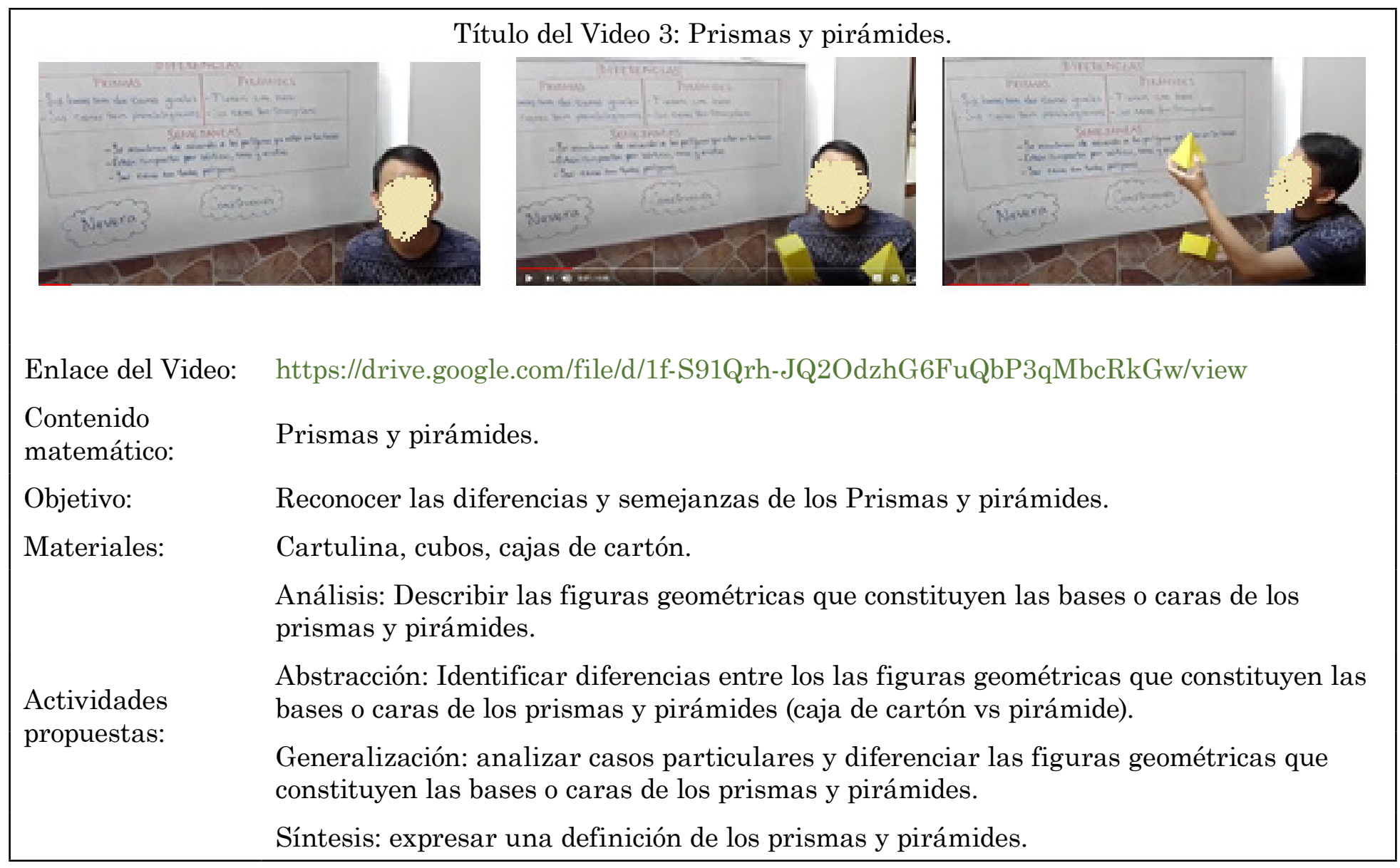

Figura 7. Descripción del contenido del video creativo de geometría: Primas y pirámides. Fuente: Elaboración propia.

El video creativo de geometría descrito en la Figura 7 aborda la temática "prismas y pirámides", el profesor en formación recurre a materiales concretos como cartulina, cajas de cartón y cuerpos geométricos prefabricados para reconstruir una situación que le permita evidenciar a los estudiantes las diferencias y semejanza de los objetos matemáticos en estudio. Las actividades propuestas en el video demandan al estudiante analizar, abstraer y generalizar las figuras geométricas planas que constituyen los prismas y las pirámides, esto mediante la comparación de las figuras geométricas utilizadas en las bases y caras laterales de los prismas en comparación con las utilizadas en las pirámides.

Una vez se consolidada la descripción del contenido de los videos creativos, se presentan las actividades diseñadas por los profesores en formación en cada una de los momentos del video: inicial, intermedio 1, intermedio 2 y final (Tabla 3). En el momento inicial, se describen las actividades que propusieron los profesores para analizar las características que satisfacen los Objetos Matemáticos (OM) en estudio (figuras geométricas, ángulos, prismas, entre otros). En el momento inicial 1, se presentan las actividades requeridas por los profesores para abstraer las características de los objetos matemáticos. En el momento inicial 2, se describen las actividades que propusieron los profesores para generalizar las características y en el momento final del video, se exponen las actividades que propusieron los profesores para sintetizar las características de los O.M en estudio. Integrar los momentos clave de un video con el proceso de formación de conceptos, permitió a los profesores de matemáticas en formación evidenciar desde actividades prácticas las relaciones matemáticas permean en objetos concretos. Además, se generan espacios de reflexión para que los estudiantes reconozcan la relación estrecha que guarda la matemática con el mundo. 
En este sentido, el objetivo del a investigación se alcanzó y evidencia las implicaciones que tiene la metodología VCG sobre la enseñanza de la matemática.

TABLA 3.

Actividades solicitadas en el contenido del video creativo de geometría

\begin{tabular}{|c|c|c|c|}
\hline \multicolumn{4}{|c|}{ Contenido del Video Creativo de Geometría } \\
\hline $\begin{array}{l}\text { Momento inicial } \\
\text { (Análisis) }\end{array}$ & $\begin{array}{l}\text { Momento intermedio } 1 \\
\text { (Abstracción) }\end{array}$ & $\begin{array}{l}\text { Momento intermedio } 2 \\
\text { (Generalización) }\end{array}$ & $\begin{array}{l}\text { Momento final } \\
\text { (Síntesis) }\end{array}$ \\
\hline $\begin{array}{l}\text { Reconocer características de } \\
\text { los objetos implementados } \\
\text { (figura, medidas). } \\
\text { Medir los lados y ángulos } \\
\text { de los triángulos. } \\
\text { Recortar figuras } \\
\text { geométricas sobre } \\
\text { cartulina. } \\
\text { Superponer figuras } \\
\text { geométricas recortadas. } \\
\text { Identifica las figuras } \\
\text { geométricas. }\end{array}$ & $\begin{array}{l}\text { Identificar características } \\
\text { que describen el } \\
\text { triángulo } \\
\text { Prescindir de las } \\
\text { características no } \\
\text { comunes del objeto } \\
\text { matemático } \\
\text { Identificar diferencias } \\
\text { entre los objetos que } \\
\text { representa los triángulos, } \\
\text { círculos, demás. }\end{array}$ & $\begin{array}{l}\text { Abordar el estudio de } \\
\text { casos particulares que } \\
\text { impliquen establecer una } \\
\text { generalización de las } \\
\text { características del objeto } \\
\text { matemático. } \\
\text { Analizar casos particulares } \\
\text { y diferenciar las } \\
\text { características de las } \\
\text { figuras/cuerpos geométricos } \\
\text { implícitas en un número } \\
\text { mayor de casos. }\end{array}$ & $\begin{array}{l}\text { Presentar las } \\
\text { características } \\
\text { invariantes } \\
\text { identificadas en una } \\
\text { expresión oral o } \\
\text { escrita } \\
\text { Presentar una } \\
\text { definición que reuna } \\
\text { las características } \\
\text { de las figuras } \\
\text { geométricas } \\
\text { estudiadas. }\end{array}$ \\
\hline
\end{tabular}

Fuente: Elaboración propia.

\section{Conclusiones}

Los resultados de esta investigación proporcionan fundamentos teóricos y una propuesta metodológica que permite a los profesores de matemáticas diseñar videos de clases prácticas que impliquen el análisis de materiales concretos. Se identificó, además, que el uso de los videos creativos como un medio didáctico favorece el aprendizaje de la geometría fundamentados en la interacción con un material concreto y un conjunto de actividades que facilitan sintetizar las características de los objetos matemáticos de la geometría.

En el contexto de la enseñanza de la geometría y el uso de videos creativos en el que se enmarca esta investigación, los resultados presentados atienden a la problemática existente ante la situación de contingencia y distanciamiento social que imposibilita realizar de forma presencial los procesos de enseñanza y aprendizaje. En línea con el objetivo trazado, se contribuye una propuesta metodológica para la elaboración de Videos Creativos de Geometría (VCG) que proporciona un conjunto de principios que permiten a los profesores de matemáticas diseñar sus propios videos creativos con el fin de mejorar la enseñanza de la geometría.

De las actividades solicitadas en el contenido de los videos creativos de geometría se identificaron que son de gran ayuda para los profesores interesados en el diseño de nuevos videos. Estas actividades están alineadas con el proceso de formación de conceptos que, en el marco de la construcción de conocimiento teórico, se requiere de cuatro actividades cognoscitivas: analizar, abstrae, generalizar y sintetizar. Se identificó que, la integración de los procesos de formación de conceptos con los momentos de los videos son un elemento fundamental en la propuesta metodológica de esta investigación. Cada momento del video representa una oportunidad para que los estudiantes construyan conocimiento teórico desde lo sensitivo con la ayuda de los materiales concretos hasta lo abstracto de los objetos matemáticos 
En suma, los videos creativos de geometría y la propuesta metodológica presentada en esta investigación constituyen una herramienta metodológica para los profesores de matemáticas interesados en la enseñanza de la geometría con base en procesos de formación de conceptos. Las fases de la metodología (VCG) conducen al profesor en la planeación, diseño y ejecución del video creativo, así también, cuenta con una descripción de videos creativos diseñados por futuros profesores con el fin de generar espacios de reflexión en cuanto a medios digitales abiertos que apoyen los procesos de enseñanza de la geometría en tiempos de pandemia. Esta investigación reúne elementos teóricos y metodológicos que son de apoyo para el diseño y ejecución de videos creativos presentados a los estudiantes como apoyo a los contenidos matemáticos del momento iniciar o finalizar el acto pedagógico.

\section{REFERENCIAS}

Ballesteros-Ballesteros, V., Rodríguez-Cardoso, Ó. y Lozano-Forero, S. (2021). Conjeturación del teorema del valor medio para derivadas: Un acercamiento desde la detección de invariantes en dispositivos móviles con GeoGebra. Cultura, Educación y Sociedad, 12(1), 19-84. https://doi.org/10.17981/cultedusoc.12.1.2021.05

Burgos, M., Beltrán-Pellicer, P. y Godino, J. D. (2020). La cuestión de la idoneidad de los vídeos educativos de matemáticas: una experiencia de análisis con futuros maestros de educación primaria. Revista Española de Pedagogía, 78(275), 27-49. https://doi. org/10.22550/REP78-1-2020-07

Braun, T. \& Lindeman, U. (may. 2004). Method adaptation, a way yo improve methodical producto development. In: D. Marjanovic, International design conferente, Dubroynik, Croatia, pp. 977-982. Available: https:/www.designsociety.org/publication/19866/ METHOD+ADAPTATION+-+A+WAY+TO+IMPROVE+METHODICAL+PRODUCT +DEVELOPMENT

Camargo, L. y Acosta, M. (2012). La geometría, su enseñanza y su Aprendizaje. Tecné, Episteme y Didaxis, 32, 4-8. https://doi.org/10.17227/ted.num32-1865

Camargo, L., Samper, C. y Perry, P. (2018). Actividad demostrativa protagonista en la clase de geometría. En A. Avila, Rutas de la Educación Matemática (pp. 176-193). Ciudad de México: SOMIDEN.

De la Fuente, M. Hernández, M. y Pra, I. (2012). El mini video como recurso didáctico en el aprendizaje de materias cuantitativas. Revista Iberoamericana de Educación a Distancia, 16(2), 177-192. https://doi.org/10.5944/ried.16.2.9911

Díaz, M. (2007). Visualización y generalización; el caso de la determinación de lugares geométricos. En, C. Dolores, G. Martínez, R. Farfán, C. Carrillo, I. López y C. Navarro, Matemática Educativa, algunos aspectos de la Socioespistemología y la visualización en el aula (pp. 207-230). México, D.F.: Días de Santos.

Francesc, P. (2020). COVID-19 y educación superior en américa latina y el caribe: efectos, impactos y recomendaciones políticas. Análisis Carolina, 36/2020, 1-15. Recuperado de: https://www.fundacioncarolina.es/wp-content/uploads/2020/06/AC-36.-2020.pdf 
Gutiérrez, A. y Jaime, A. (2012). Reflexiones sobre la enseñanza de la geometría en primaria y secundaria. Tecné, Episteme y Didaxis, (32), 55-70. http://dx.doi.org/10.17227/ ted.num32-1859

Hernández, R., Fernández, C. y Baptista, P. (2014). Metodología de la investigación. (6 ed.). México D.F.: McGraw-Hill.

Morales, L., Guzmán, T. y Tecnología Educativa. (noviembre, 2015). El vídeo como recurso didáctico para reforzar el conocimiento. Conferencia presentada en las Memorias del Encuentro Internacional de Educación a Distancia, Univesidad de Guadalajara, Guadalajara, México. http://dx.doi.org/10.13140/RG.2.1.3144.7521

Moreno, F. (2015). La utilización de los materiales como estrategia de aprendizaje sensorial en infantil. Opción, 31(Especial 2), 772-789. Disponible en https://www.produccioncientificaluz.org/index.php/opcion/article/view/20416

Moreno-Armella, L. y Elizondo, R. (2017). La Geometría al encuentro del aprendizaje. Educación Matemática, 29(1), 9-36. http://dx.doi.org/10.24844/EM2901.01

Moreno-Correa, S. M. (2020). La innovación educativa en los tiempos del Coronavirus. Salutem Scientia Spiritus, 6(1), 14-26. Disponible en https://revistas.javerianacali. edu.co/index.php/salutemscientiaspiritus/article/view/2290

Planas, N. (2006). Modelo de análisis de videos para el estudio de procesos de construcción de conocimiento matemático. Educación Matemática, 18(1), 37-72. Disponible en http://www.revista-educacion-matematica.com/revista/vol18-1/

República de Colombia. Presidencia de la República. (13 de mayo de 2020). Por el cual se dictan medidas relacionadas con el calendario académico para la prestación del servicio educativo, en el marco del Estado de Emergencia Económica, Social y Ecológica. [Decreto 660]. Diario Oficial No. 51.313. Recuperado de https://www.mineducacion. gov.co/1759/articles-397834_archivo_pdf.pdf

República de Colombia. Presidencia de la República. (28 de mayo de 2020). Por el cual se imparten instrucciones en virtud de la emergencia sanitaria generada por la pandemia del Coronavirus COVID-19, y el mantenimiento del orden público. [Decreto 749]. Diario Oficial No. 51.328. Recuperado de https://dapre.presidencia.gov.co/normativa/ normativa/DECRETO\%20749\%20DEL\%2028\%20DE\%20MAYO\%20DE\%202020.pdf

Sandoval, I., Cagua, B., Álvarez, F., Molina, M. y Estrada P. (julio, 2018). El Video Educativo Como Estrategia Metodológica en la Enseñanza Universitaria. Presentado en el 16th International Multi-Conference for Engineering, Education, and Technology: "Innovation in Education and Inclusion", LACCEI, Lima, Peru. http://dx.doi. org/10.18687/LACCEI2018.1.1.286

UNESCO. IESALC (2020). COVID-19 y educación superior: De los efectos inmediatos al día después. Análisis de impactos, respuestas políticas y recomendaciones. Caracas: UNESCO IESALC. Recuperado de http://www.iesalc.unesco.org/wp-content/ uploads/2020/05/COVID-19-ES-130520.pdf 
Velasco, A. M., Montiel, S. y Ramírez, S. (2018). Los videos educativos como herramienta disruptiva para apoyar el proceso de aprendizaje de algoritmos de resta y multiplicación en estudiantes de segundo grado de primaria. Revista Educación, 42(2), 1-20 Disponible en https://revistas.ucr.ac.cr/index.php/educacion/article/view/24236

Jonathan Cervantes-Barraza. Es licenciado en matemáticas de la Universidad del Atlántico (Colombia). Profesor de matemáticas en educación primaria. Realizó estudios de maestría en Ciencias del Área de Matemáticas Educativa de la Uni (México). Actualmente es estudiante del último semestre en la Especialidad en Matemáticas Educativa por la misma universidad. Investiga sobre la argumentación y prueba matemática, formación, geometría y ecuaciones diferenciales. Ha publicado artículos en revistas como Bolema (Brasil), Educación Matemática (México), PNA School Science and Mathematics Journal (USA), ha participado como ponente en importantes eventos científicos. https://orcid. org/0000-0002-7000-4977 